\title{
TRASCENDENCIA DE LA RESIDENCIA HABITUAL EN LAS CRISIS FAMILIARES EN EL DERECHO EUROPEO. COMENTARIO DE LA SENTENCIA DE 24 DE JULIO DE 2019 DE LA SECCIÓN SEGUNDA DE LA AUDIENCIA PROVINCIAL DE GIRONA*
}

\author{
TRANSCENDENCE OF THE HABITUAL RESIDENCE IN \\ FAMILY CRISIS IN THE EUROPEAN LAW. DISCUSSION OF \\ THE JUDGEMENT OF JULY 24, 2019 SECOND SECTION OF \\ THE PROVINCIAL COURT OF GIRONA
}

\author{
LuCAS ANDRÉs PÉREZ MarTíN** \\ Profesor Contratado Doctor de Derecho internacional privado \\ Universidad de Las Palmas de Gran Canaria \\ ORCID ID: 0000-0001-6340-0528
}

Recibido: 16.12.2019 / Aceptado: 10.01.2020

DOI: https://doi.org/10.20318/cdt.2020.5212

\begin{abstract}
Resumen: La sentencia de la Sección Segunda de la Audiencia Provincial de Girona de 24 de julio de 2019 nos permite destacar la trascendencia que en las relaciones internacionales entre privados tiene la residencia habitual de las partes en el momento en el que surge el conflicto. En este caso, la residencia habitual en España en el momento del divorcio de dos cónyuges de nacionalidad francesa, casados en Francia, y con propiedades en España y en Francia, provocó, en aplicación de la normativa europea que aquí analizaremos, la competencia judicial de los tribunales españoles, y la aplicación del derecho español. No dejaremos pasar la ocasión para exponer que argumentación y la terminología empleada en la resolución podrían hacer sido más exactas y correctas.
\end{abstract}

Palabras clave: divorcio contencioso internacional, residencia habitual, nacionalidad, ámbito de aplicación.

Abstract: The sentence of the Second Section of de Provincial Court of Girona of July 24, 2019 allows us to highlight the importance of the habitual residence for the parties in the international relations between private parties at the moment when the conflict arises. In this case, the habitual residence in Spain caused at the time of the divorce of two spouses of French nationality, married in France, and with properties in Spain and France, and due to the application of the European regulations will be analyzed here, the judicial competence of the Spanish courts and the completion of the procedure through the Spanish legislation. We will not miss the occasion to highlight that the argumentation and the terminology used in the final decision could have been more accurate and correct.

Keywords: international contentious divorce, habitual residence, nationality, scope of application.

* Este trabajo ha sido realizado en el marco del Proyecto de Investigación I+D+I: "Obstáculos a la movilidad de personas en los nuevos escenarios de la UE”, financiado por el Ministerio de Economía y Competitividad, Dirección General de Investigación Científica y Técnica, con la referencia DER2017-86017-R.

**1ucas.perez@ulpgc.es 
Sumario: I. Los hechos y el derecho, Derecho internacional privado y mucho más. II. La cuestión esencial, nacionalidad o residencia habitual como criterios: 1. Alegaciones de las partes. 2. La residencia habitual, criterio principal en el Derecho europeo en crisis familiares. 3. Delimitación de la residencia habitual en crisis familiares internacionales y de menores. III. Acierto en la valoración de los Tribunales españoles: 1. Correcta valoración del lugar de residencia habitual de las partes. 2. Competencia judicial española y el cambio de residencia de la hija. 3. La ley aplicable, la española, sistema plurilegislativo. IV. Derecho internacional privado, argumentación y cuestiones terminológicas, la importancia de la exactitud: 1. El ámbito de aplicación, lo que abunda a veces daña. 2. El derecho aplicable, la importancia de la argumentación 3. Control de oficio de la competencia y la perpetuatio fori. V. Conclusiones, algo más que la residencia habitual.

\section{Los hechos y el derecho, Derecho internacional privado y mucho más}

1. La sentencia de la Sección Segunda de la Audiencia Provincial de Girona de 24 de julio de $2019^{1}$, que necesariamente ha de ser analizada junto a la del Juzgado de Primera Instancia e Instrucción $\mathrm{n}^{\circ} 7$ de Figueras que revisa y a la que se remite en el aspecto fundamental que afecta al Derecho internacional privado ${ }^{2}$, nos ofrecen diversos aspectos de interés para la disciplina. En un procedimiento de divorcio de ámbito internacional, surge la controversia sobre dónde conocer del asunto y qué ley aplicar toda vez que las partes tienen destacadas relaciones con dos países concretos, Francia y España, y porque una vez iniciado el proceso en España, la demandante se traslada con su hija a Francia. La primera cuestión esencial, atendiendo al fondo del asunto, es la de la trascendencia de la residencia habitual o de la nacionalidad de las partes en el momento del inicio del proceso para localizar la competencia judicial internacional y establecer la ley aplicable para resolver el conflicto. En segundo lugar, la importancia que tiene para concretar la competencia y la ley aplicable el traslado de la demandante con su hija, y no del demandado, una vez iniciado el proceso, de España a Francia. Finalmente, ambas resoluciones también muestran interés para el jurista en relación a la trascendencia de la correcta argumentación y uso de la terminología de Derecho internacional privado, no siempre el más aconsejable por parte de los operadores jurídicos ${ }^{3}$.

2. Antes de entrar en los aspectos de fondo de la sentencia es imprescindible detenernos en los hechos del proceso, toda vez que estos, lógicamente, y más en este caso, influyen directamente en la regulación aplicable para resolver el conflicto ${ }^{4}$. Estamos ante un matrimonio de ciudadanos franceses casados en Francia el 25 de junio de 1994. Los efectos económicos de su matrimonio son regulados por el régimen de separación de bienes del Derecho francés ${ }^{5}$. El matrimonio ha tenido tres hijos, nacidos en 1993, 1997, y 2002. Residen en Figueras desde hace muchos años previamente a que en 2016 surja el conflicto familiar y la madre interponga la demanda de divorcio y de medidas respecto a los hijos y a ella

\footnotetext{
${ }^{1}$ ST AAPP Girona 24 julio 2019, ECLI:ES:APGI_2019_1064.

${ }_{2}^{2}$ Sentencia del Juzgado de Primera Instancia e Instrucción $n^{0} 7$ de Figueras, exclusivo de violencia sobre la mujer, de 14 de diciembre de 2018, en los Autos de divorcio contencioso 69/2016. Sin publicación oficial en los repertorios habituales agradecemos a la Jueza responsable del Juzgado, María Teresa Ferrer Costa, el envío de la misma y su disponibilidad para ello. Tal y como veremos en el presente trabajo, el desarrollo de la fundamentación vinculada al Derecho internacional privado lo hace esta sentencia, y a ella se remite la de segunda instancia, por lo que sin esta primera resolución hubiese sido imposible el comentario de la segunda.

${ }^{3}$ Demostrando la validez de aquella frase que tanto citamos en nuestra actividad en la disciplina, que señala que el Derecho internacional privado es la materia amada por quienes la investigan, temida por quienes la estudian y olvidada por quienes la aplican. Trabajamos en una "rama del saber realmente compleja, profundamente técnica que requiere una ingente dosis de esfuerzo para su correcta aprensión y aplicación", vid. A. L. Calvo Caravaca, y J. Carrascosa González, Derecho Internacional Privado, Vol. I, Madrid, 18 a edición, Comares, 2018, prólogo, parágrafo 3. A esto se le suma su cada vez menor valor en el currículum académico del Grado en Derecho e incluso su desgraciada desaparición en muchos programas del Máster de Acceso a la Abogacía.

${ }^{4}$ Para ello contamos con las dos resoluciones, la de primera y la de segunda instancia, y los hechos que estas describen. No señalan con exactitud algunos de los años en los que ocurren los hechos, lo que hubiese sido deseable en aras de la mayor concreción del relato, pero de la descripción que exponemos podemos derivar que contamos con los datos básicos de los mismos para poder realizar un adecuado análisis del sentido final de las resoluciones.

${ }^{5} \mathrm{Cf}$. FJ $5^{\mathrm{o}}$ de la sentencia de apelación, página 7.
} 
misma. El patrimonio del esposo y padre de los hijos es amplio, valorándole en no inferior a los once millones de euros, sin contar con participaciones en sociedades mercantiles y otros bienes ${ }^{6}$. La esposa, desde casi el principio del tiempo de vigencia del matrimonio, no ha trabajado en profesión remunerada externa al trabajo desarrollado en el cuidado del hogar familiar y de los hijos. En el momento en el que surge el conflicto, dada la situación precaria en la que queda la esposa, sin patrimonio propio, se traslada a Francia por motivos económicos para vivir en casa de una hermana ${ }^{7}$. El esposo ha seguido residiendo en España durante todo el procedimiento ${ }^{8}$.

3. Como vemos, el procedimiento tiene varias aristas de gran interés para el Derecho internacional privado y para las partes, por lo que hablamos de Derecho internacional privado y mucho más. En primer lugar, un matrimonio de larga duración con un esposo que ha acumulado un patrimonio privativo elevado y una esposa que no lo ha hecho y que queda en situación precaria tras el divorcio, con tres hijos, dos mayores de edad y una cercana a los 18 años, y dos de ellos en periodo de formación aún'. Clara relación con dos Estados miembros de la Unión Europea. España, en la que en el momento de la interposición de la demanda desarrollan su vida desde hace años ${ }^{10}$ y el marido tiene propiedades. Y también con Francia, del que son nacionales, donde se casaron e iniciaron la vida en común, donde tiene bienes el marido, y a donde se han trasladado la demandante y sus dos hijos tras la interposición de la demanda en España. Las sentencias establecieron la patria potestad conjunta y la guarda y custodia de la hija menor de edad a la madre sin un régimen de visitas concreto ${ }^{11}$. El pago de los gastos extraordinarios se asumiría el $75 \%$ por parte del padre y el $25 \%$ por parte de la madre. Finalmente se determinaron cuantías de pensiones alimenticias y compensatorias elevadas, si bien menores en la apelación que en la instancia ${ }^{12}$. Así pues, analizaremos la determinación del lugar de residencia habitual de la familia y su importancia para localizar la competencia judicial internacional y establecer el derecho aplicable. Las alegaciones del demandado sobre la aplicación del criterio de nacionalidad o del traslado de la residencia de la menor tras la interposición de la demanda. Por último, aspectos vinculados a la propia redacción de las dos sentencias analizadas. Todo ello teniendo en cuenta la trascendencia que para el proceso tiene el elevado patrimonio del esposo y la precaria situación de la esposa tras el divorcio, en contraste con su vida anterior ${ }^{13}$.

${ }^{6} \mathrm{Cf}$. $\mathrm{FJ} 3^{\circ}$ de la sentencia de apelación, que señala que tributa en los dos países, Francia y España, lo que dificulta el cálculo exacto de los bienes que posee. Llega a cifrar el patrimonio en 12 millones de euros.

${ }^{7} \mathrm{Cf}$. $\mathrm{FJ}^{\circ}$ de la sentencia de apelación. La mujer se vio obligada a trasladarse a Francia, inicialmente a casa de una hermana, por no disponer de medios económicos para mantener la vivienda española, toda vez que únicamente contaba con la pensión asignada provisionalmente en el procedimiento de divorcio. En el momento de la resolución de segunda instancia vivía en una vivienda de alquiler en Francia.

${ }^{8} \mathrm{Cf}$. $\mathrm{FJ} 2^{\circ}$ de la resolución de segunda instancia, última frase.

${ }^{9} \mathrm{Cf}$. $\mathrm{FJ} 4^{\mathrm{o}}$ de la resolución de instancia y $5^{\circ}$ de la sentencia de apelación. La menor ya tenía 17 años y el hermano 22 y seguía formándose en cursos de cocina en Francia.

${ }^{10} C f$. FJ $1^{\circ}$ de la resolución de instancia, p. 5. La resolución solo señala que la familia entera se encontraba viviendo en España, ya que habían fijado el domicilio familiar "desde hace años antes de producirse la ruptura matrimonial". Para el análisis de las circunstancias de hecho del caso hubiese sido deseable saber exactamente desde hacía cuántos años, en qué momento trasladaron su residencia de Francia a España, pero nadie impugna el hecho ni la circunstancia de la larga permanencia de la familia en España antes del divorcio. No sabemos, desde 1994, año en el que se casaron en Francia, cuándo empezaron a residir en España, pero debió ser poco tiempo después de la celebración del matrimonio, atendiendo al relato de hechos de las dos resoluciones y a que en las alegaciones de las partes que estas citan en ningún momento se puso en cuestión dicho hecho.

${ }^{11} \mathrm{Cf}$. $\mathrm{FJ} 4^{\circ}$ de la resolución de instancia. La menor ya tenía 17 años, y manifestaba su voluntad de seguir residiendo con la madre y no querer tener contacto con el padre.

${ }^{12} C f$. fallos de ambas resoluciones. En primera instancia, pensión alimenticia a la hija menor de edad de 1.500 euros mensuales, y al hijo mayor de edad de 1.500 euros mensuales durante tres meses. A la esposa, pensión compensatoria de 8.000 euros mensuales durante 10 años. Manteniendo los periodos la Audiencia Provincial las rebajó a 1.200 euros a los hijos y 6.000 euros a la esposa.

${ }^{13} C f$. FJ $3^{\circ}$ a 5 de la sentencia de apelación. En el análisis de las circunstancias familiares para fijar la pensión compensatoria de la esposa cita expresamente que estamos ante una familia "económicamente privilegiada que ha mantenido un nivel de vida acorde con tal estado", que "disponían de una importante fortuna de origen familiar" y los hijos "de todo aquello que es consecuencia de la abundancia", citando incluso "viviendas de alto standing, lingotes de oro, viajes suntuosos en aviones privados, Mexico, Dubai, Guadalupe, Grecia, Cavo Verde, dos embarcaciones, motos acuáticas, vehículos de lujo (Maseratti, Mercedes...), otros vehículos (quads, karting, motocicletas y ciclomotores diversos, bicicletas eléctricas)”. 


\section{La cuestión esencial, nacionalidad o residencia habitual como criterios}

\section{Alegaciones de las partes}

4. La demandante interpone la demanda en España, en su nombre, y solicitando además del divorcio pensiones alimenticias para dos hijos de 22 y 17 años y compensatoria para ella misma. En ningún momento el relato de las sentencias analizadas señala que realice otra afirmación diferente sobre el lugar competente para conocer del divorcio y, lo debemos dar por hecho, la ley aplicable al mismo. Así pues, considera que la competencia es España y la ley aplicable la española. El motivo es evidente, la familia, en dicho momento, tiene la residencia habitual en España desde hace muchos años, y por ello, aunque no tengamos el documento de demanda, a buen seguro acude a dicho argumento para interponer la demanda en España aplicando el derecho español. Tras interponer la demanda la madre cambia su residencia habitual a Francia, y se va a vivir, por motivos económicos, con una hermana, sin que considere que ello deba afectar a la competencia por aplicación de los artículos 10 a 12 del Reglamento Bruselas II bis.

5. El demandado, en la primera instancia, cuestiona la competencia judicial internacional del órgano judicial español por el cambio de residencia de la hija menor a Francia tras la interposición de la demanda, citando que, por este hecho, el interés de la mejor reside en que la competencia sea la de los tribunales franceses ${ }^{14}$. Por otro lado, la resolución de apelación, respecto a nuestra materia de Derecho internacional privado ${ }^{15}$, señala que el demandado muestra su disconformidad con la sentencia de instancia, y cambia su alegación principal y, recurriendo a la necesidad de pronunciamiento de la Audiencia Provincial por tratarse de una cuestión de orden público, alega que los tribunales competentes son los franceses ya que el procedimiento lo dirimen dos personas de nacionalidad francesa ${ }^{16}$, volviendo a solicitar por ello la declaración de falta de competencia judicial de los tribunales españoles.

\section{La residencia habitual, criterio principal en el Derecho europeo de crisis familiares}

6. Así pues, nos encontramos ante el ya clásico debate sobre la residencia habitual y la nacionalidad como criterios de localización de la competencia judicial internacional y como puntos de conexión de la ley aplicable. Sobre esta materia mucho se ha escrito, y no entraremos en detalle en ella por no ser el objeto del presente trabajo ${ }^{17}$. Al respecto, y si bien nos detendremos en el epígrafe III en el motivo por el cual la regulación de la Unión Europea es la aplicable al presente caso, únicamente dejaremos citado

\footnotetext{
${ }^{14}$ Según lo señalado en el FJ $1^{\circ}$ de la sentencia de instancia, p. 5, en el escrito de contestación a la demanda el demandado aceptó la competencia española, y la cuestionó durante el proceso con posterioridad por el cambio de residencia de la menor alegando que estamos ante una cuestión de "orden público". Aunque volveremos a este hecho en el epígrafe III.2, tampoco sería extraña esta contradicción respecto a la posición inicial en el proceso y a la posterior, si la hija hubiese cambiado su lugar de residencia tras la contestación a la demanda o si él lo conoció en dicho momento, dato concreto que no conocemos por no constar en las sentencias analizadas.

${ }^{15}$ También impugna el recurrente la determinación de las cuantías de las pensiones alimenticias y compensatorias en el ámbito del Derecho de familia.

${ }^{16} C f$. FJ $2^{\circ}$ de la sentencia de apelación. Únicamente señala en un breve fundamento la alegación de la nacionalidad de las partes, en ningún momento el cambio de residencia habitual de la hija tras la presentación de la demanda. A pesar de que no contemos con el escrito de interposición del recurso de apelación -fue solicitado, pero no pudimos obtenerlo-, el hecho de que no se recoja la argumentación del cambio de residencia nos conduce a considerar que se cambió de argumento ante la falta de éxito del empleado en primera instancia, que tal y como expondremos posteriormente, tenía más visos de prosperabilidad, si bien estimamos que la resolución final fue acertada

${ }^{17} \mathrm{Al}$ respecto, vid. J. M., Espinar Vicente, La nacionalidad y la extranjería en el sistema jurídico español, Madrid, Civitas, 1994; A. Rodríguez Benot, "El criterio de conexión para determinar la ley personal: un renovado debate en Derecho Internacional Privado", $C D T$, marzo 2010, Vol. 2, n 1, pp. 186 a 202; D. CAVERs, "Habitual residence: a useful concept?", $21 \mathrm{Am} . \mathrm{U}$. L. Rev. 475 (1971-1972), pp. 475 a 493; P. Rogerson, "Habitual residence: the new domicile?", Int'l \& Comp. Law Q., 2000, Vol. 49, n 1, pp. 86 a 107. Lo desarrollamos con mayor detenimiento, y a dicho trabajo nos remitimos, en L. A. Pérez Martín, "Propuesta de un concepto europeo de residencia habitual en el derecho de familia internacional", AEDIPr, t. XVIII, $2018, \mathrm{pp}$. 469 a 494.
} 
que, respecto a la competencia judicial internacional, el Reglamento Bruselas II Bis ${ }^{18}$, aplicable al presente supuesto, al que ha seguido en su contenido la $\mathrm{LOPJ}^{19}$, regula la competencia judicial internacional en materia de divorcio, y tiene la finalidad de vincular el proceso a los lugares en los que los cónyuges tengan una mayor relación. El mismo establece en su artículo 3 una serie de foros que son elegibles alternativamente por las partes que inician el proceso, y por ello todos admisibles en el presente supuesto, sin que la normativa establezca que uno tenga preferencia frente a los otros. Estos foros se regulan en su apartado 1 a y b. En el a, en primer lugar, admite el foro de "la residencia habitual de los cónyuges si es conjunta", y también, "la última residencia habitual de ambos cónyuges si aún uno de ellos reside en dicho lugar". En tercer lugar, "la residencia habitual del demandado". También se admiten como foros competentes en caso de traslado de uno de los cónyuges, la "residencia del demandante si ha residido en ella al menos durante un año antes de interponer la demanda", o la "residencia del demandante si es el país de su nacionalidad y ha residido en ella al menos durante seis meses antes de interponer la demanda". Finalmente, en la letra b del artículo, se admite también como foro competente el del Estado de "la nacionalidad de ambos cónyuges" si la nacionalidad de ambos es la misma.

7. El legislador ha tenido como principal finalidad la de vincular el proceso al lugar en el que se desarrolló la vida en común del matrimonio si es posible y no ha pasado mucho tiempo desde que la relación conyugal finalizó, por lo que la residencia habitual común es el primer criterio que considera la norma ${ }^{20}$. Y este aspecto ha de tenerse muy en cuenta en todos los procedimientos de divorcio internacional. En caso de que haya pasado mucho tiempo el siguiente criterio es el de la residencia habitual posterior de las partes consideradas por separado. Esos posibles foros los establece en primer lugar, en la letra a, dándole gran trascendencia, por lo que señalamos que la residencia habitual es el principal criterio fijado por la legislación europea ${ }^{21}$. Si bien también es aplicable alternativamente, solamente en un segundo momento entra en juego la nacionalidad de las partes y únicamente si son nacionales del mismo país ${ }^{22}$. Este criterio es también aplicable para establecer el foro competente para conocer el procedimiento si se da y la parte demandante decide aplicarlo, pero creemos claro y evidente que el criterio principalmente señalado por el legislador es el de la residencia habitual.

8. En la regulación de la ley aplicable a las crisis familiares internacionales nos encontramos ante un esquema algo similar respecto a la trascendencia de la residencia habitual como punto de conexión. El Reglamento Roma III ${ }^{23}$ también ha desplazado a la nacionalidad, considerándolo un criterio claramente secundario. En caso de falta de acuerdo, los dos primeros criterios, de cuatro en cascada, estos no alternativos, remiten a la residencia habitual ${ }^{24}$. Los dos primeros son los de la "ley del Estado de la residencia habitual de ambos cónyuges en el momento de la interposición de la demanda", y si los

\footnotetext{
${ }^{18}$ Reglamento (CE) n ${ }^{\circ}$ 2201/2003 del Consejo de 27 de noviembre de 2003 relativo a la competencia, el reconocimiento y la ejecución de resoluciones judiciales en materia matrimonial y de responsabilidad parental, por el que se deroga el Reglamento $(\mathrm{CE}) \mathrm{n}^{\mathrm{o}} 1347 / 2000$, DOUE L338/1 de 23 de diciembre de 2003.

${ }^{19}$ La reforma de la LOPJ de julio de 2015 estuvo claramente inspirada en la legislación europea. Al respecto, vid. C. EsPLUgues Mota, J. L. Iglesias Buhigues, G. Palao Moreno, Derecho Internacional Privado, Valencia, 13 a edición, Tirant lo Blanch, 2019, pp. 114 y 115, o F. J. Garcimartín Alférez, Derecho Internacional Privado, Pamplona, 4a edición, Civitas, 2017 , p. 88.

${ }^{20}$ Y no solo el principal por importancia y por número de foros alternativos que establece, sino también en el hecho porcentual de que, de siete foros, seis están vinculados a la residencia habitual, y solo uno de ellos a la nacionalidad de ambas partes. Los detalla, por ejemplo, P. Orejudo Prieto de los Mozos, "Diez años de aplicación e interpretación del Reglamento Bruselas I bis sobre crisis matrimoniales y responsabilidad parental (análisis de los aspectos de competencia judicial internacional)", $L a$ Ley UE, núm. 21, 2014, p. 17.

${ }^{21}$ La trascendencia de este criterio de la residencia habitual desde una perspectiva práctica, lo que excede del contenido de nuestro estudio, la analiza, por ejemplo, O. D. LudeÑa Benítez, "El derecho de familia de la Unión Europea: cuestiones de cooperación jurídica comunitaria entre los Estados Miembros”, Revista Jurídica de Castilla y León, Núm. 32, enero 2014, pp. 17 a 19.

${ }^{22}$ Tal y como ocurría en el presente supuesto, lo que analizaremos en el epígrafe III de nuestro trabajo.

${ }^{23}$ Reglamento (UE) no 1259/2010 del Consejo de 20 de diciembre de 2010 por el que se establece una cooperación reforzada en el ámbito de la ley aplicable al divorcio y a la separación judicial, DOUE L343/10 de 29 de diciembre de 2010. La modificación de la legislación española le quita trascendencia práctica al hecho de que el reglamento se haya aprobado por cooperación reforzada.

${ }^{24} C f$. art. 8 del Reglamento.
} 
cónyuges no residen en el mismo lugar, el segundo criterio es "ley del Estado en el que los cónyuges hayan tenido su última residencia habitual, siempre que el período de residencia no haya finalizado más de un año antes de la interposición de la demanda, y que uno de ellos aún resida allí en el momento de la interposición de la demanda" ${ }^{25}$. Solo el tercer criterio nos remite a la nacionalidad, y también únicamente si es común de los cónyuges en el momento en el que se interpone la demanda. El cuarto establece que se aplicará la ley del foro competente ${ }^{26}$. Así pues, vemos que la residencia habitual de las partes si es común o está aún relacionada con la vida en común del matrimonio es el punto de inicial conexión para establecer la ley con la que se regulará la crisis familiar, teniendo la nacionalidad una trascendencia mucho menor $^{27}$.

\section{Determinación de la residencia habitual en crisis familiares internacionales y en menores}

9. Ninguno de los Reglamentos define la residencia habitual de las partes ni establece criterios para concretarla ${ }^{28}$. La doctrina ha expuesto que esta es una posición voluntaria del legislador, que de forma premeditada ha huido de cerrar el concepto, en la intención de que sean los tribunales los que definan, caso a caso, y siempre teniendo en cuenta el ámbito del derecho regulado, cuáles son los criterios para establecer esta residencia habitual de las partes afectadas, que variarán atendiendo al tipo de litigio de que se trate y del tipo de intereses que regule, siendo distinto el concepto para derecho contractual, de familia, menores o sucesorio ${ }^{29}$. Opinamos que esta situación debería cambiar, y que sería positivo aportar una definición concreta de la residencia habitual de las partes que diese seguridad jurídica a los operadores jurídicos, y aunque no nos detengamos aquí en el desarrollo de esta idea por no ser objeto del presente estudio ${ }^{30}$, sí que creemos que debemos definir en este momento sus aspectos básicos para poder afrontar si la determinación de la residencia habitual de las partes en la resolución analizada fue la correcta. En primer lugar debemos señalar que nos encontramos ante un concepto autónomo de Derecho europeo $^{31}$, lo que supone que tiene consideración propia en este derecho de ámbito supranacional diferenciado de la propia consideración que del mismo puedan tener los sistemas jurídicos de los distintos

${ }^{25}$ Destacan la seguridad jurídica y el acierto de establecer el punto de conexión de la residencia habitual en el reglamento, A. Calvo Caravaca y J. Carrascosa GonzÁlez, "La ley aplicable al divorcio en Europa. El futuro reglamento Roma III", CDT, marzo 2009, Vol. 1, No 1, pp. 59 a 61, y B. CAmpuZano Díaz, "El Reglamento (UE) No 1259/2010 de 20 de diciembre de 2010, por el que se establece una cooperación reforzada en el ámbito de la ley aplicase al divorcio y a la separación judicial", Revista de Derecho Comunitario Europeo, núm. 39, mayo/agosto 2011, pp. 575-578.

${ }^{26}$ Las partes se pueden poner de acuerdo en esos cuatro criterios (art. 5). Vid. A. Rodríguez Benot (Director), Derecho Internacional Privado, Madrid, Tecnos, 2018, pp. 220 a 222.

${ }^{27}$ Estudiamos con más detenimiento ambos reglamentos, y la trascendencia de la residencia habitual en el todo el derecho de familia europeo, y nos remitimos a dicho trabajo para un mayor desarrollo de esta idea, en L. A. Pérez Martín, "Determinación y trascendencia de la residencia habitual en las crisis familiares internacionales", en M. GuZMÁn ZaPATER y M. HERRÁNZ Ballesteros, Crisis matrimoniales internacionales, y sus efectos. Derecho español y de la Unión Europea, Valencia, Tirant lo Blanch, 2018, pp. 929 a 940.

${ }^{28}$ Sí lo hizo el Reglamento de sucesiones en sus considerandos 23 y 24. Al respecto, vid. J. Carrascosa GonzÁlez, "Reglamento sucesorio europeo y residencia habitual del causante", $C D T$, marzo 2016, Vol. 8, nº1, pp. 47-75; o L. A. Pérez MarTín, "Efectos del Reglamento europeo de sucesiones en las sucesiones intestadas en situaciones de residencia temporal alternativa en diferentes países", en H. Mota/, M.R. Guimaraes, Autonomia e heteronomia no direito da familia e no direito das sucesoes, Oporto, Almedina, 2018, 537 a 554.

${ }^{29}$ Esta opinión se observa tanto en la doctrina española como la europea, por ejemplo, vid. en España a J. CARRASCOSA GoNZÁLEZ, "Reglamento sucesorio europeo y residencia habitual del causante", $C D T$, marzo 2016, Vol. 8, №1, p. 62; o en Italia A. DAVì y A. ZANOBETTI, "Il nuovo diritto internazionale privato delle successioni nell'Unione europea”, CDT, octubre 2013, Vol. 5, N ${ }^{\circ}$ 2, p. 30; o centrado en las Convenciones de la Conferencia de La Haya, en M. ZHANG, "Habitual Residence v. Domicile: A Challenge Facing American Conflicts of Law", Maine Law Review, 2018, Vol 70, №2, p. 177.

${ }^{30}$ La defendimos detalladamente, y a dicho texto nos remitimos en L. A. PÉrez Martín, "Propuesta de un concepto europeo de residencia habitual...", op. cit., pp. 469 a 494. También es de la misma opinión, por ejemplo, M. A. Gandía SelLENS.: "La responsabilidad parental y la sustracción de menores en la propuesta de la Comisión para modificar el RB II Bis: algunos avances, retrocesos y ausencias", $A E D I P r$, T. XVII, 2017, p. 814.

${ }^{31}$ Así lo ha establecido la Jurisprudencia del TJUE, por ejemplo, en la Sentencia 22 de diciembre de 2010, caso Mercredi Asunto C-497/10, ECLI:EU:C:2010:829. También al respecto, vid. S. SÁnchEz Lorenzo, "El principio de coherencia en el Derecho internacional privado europeo", REDI, Vol. 70/2, 2018, pp. 30 у 31. 
Estados miembros. Y este concepto, que la Jurisprudencia del TJUE define como "el centro de vida e intereses" de la persona, se podría definir desde una perspectiva general como el lugar en el que, atendiendo al criterio de proximidad, teniendo una relación física de permanencia voluntaria con un Estado con estabilidad, la persona está integrada en él, ya que mantiene objetivos lazos personales con dicho Estado, centrados en su vida en familia, vivienda habitual o patrimonio, así como vínculos profesionales y sociales en su caso ${ }^{32}$.

10. Una vez expuesta la definición general de residencia habitual debemos establecer qué criterios tener en cuenta para determinarla en las situaciones de divorcio internacional como la resuelta en el presente procedimiento. Hay que buscar determinar si existe una verdadera residencia habitual familiar común, y dónde está de manera real, fáctica y efectiva. Cuál es el lugar en el que se encuentran los intereses comunes de la pareja en el momento de la disolución de la misma, o en los últimos momentos de la vida en común. Para ello se deben analizar el tiempo y el lugar de residencia de los cónyuges en el mismo lugar mientras el matrimonio desarrollaba su convivencia, la existencia de hijos unidos a la del domicilio habitual de los mismos junto a uno o a los dos progenitores. Un tercer factor es el posible motivo de la vida de la pareja en lugares diferentes y la razón de los traslados. Otros criterios menos importantes son la nacionalidad de todos los miembros de la familia, el idioma común que hablan unido quizás al lugar de residencia, y sus lazos personales y familiares con otras personas, así como los intereses patrimoniales de la pareja ${ }^{33}$.

11. Y toda vez que también deberemos analizar la residencia habitual de la hija menor de las partes $^{34}$, señalaremos que, respeto a la residencia de la misma, se debe tener muy en cuenta su edad, desarrollo personal y formativo, su escolarización y actividades extraescolares, el tiempo y el lugar de residencia en los distintos lugares en los que haya vivido, y en concreto en el momento en el que se produce el conflicto relativo a la relación parental. Deben analizarse los motivos y tiempos de sus traslados, su edad, madurez, y vinculada a ella su mayor o menor relación con ambos padres o solo con uno de ellos. También se deben valorar sus lazos familiares con otras personas que no sean sus padres y su entorno social, así como los amigos con los que comparte su vida. Otros factores a valorar son su nacionalidad y el idioma en el que se expresa si bien siempre se han valorado en un segundo lugar. También se debe tener en cuenta la opinión de la menor si tiene suficiente madurez, lo que ocurría en este supuesto, así como las viviendas en las que ha estado y los acuerdos anteriores que hayan existido entre los progenitores ${ }^{35}$.

${ }^{32}$ Todo ello considerado en el momento de la crisis familiar analizada, lo que es trascendental en el presente supuesto, sin que puedan ser tenidos en cuenta desplazamientos fraudulentos de las partes o realizados sin acuerdo alguno entre ellas, según lo recogido en la STJUE de 9 de octubre de 2014, caso $M$, Asunto C-376/14, ECLI:EU:C:2014:2268.

${ }_{33}$ Pocos han sido los trabajos que han afrontado la tarea de concretar estos criterios en el caso de divorcios internacionales han afrontado este mismo análisis respecto a los criterios o indicios a tener en cuenta en estos casos. Uno especialmente detallado, vid. J. CARRAscosa GonzÁLez, "La ley aplicable a la separación judicial y al divorcio en defecto de elección por los cónyuges. Análisis del artículo 8 del Reglamento 1259/2010 de 20 de diciembre 2010", CDT, marzo 2012, Vol. 4, № 1, p. 74. En relación a la pareja y los menores, vid. T. VivatVaraphol, "Back to basics: determinig a child's habitual residence in International child abduction cases under the Hage Convention", 77 Fordham L. Rev, 3325, 2008-2009, pp. 3325 a 3369.

${ }^{34}$ El Reglamento Bruselas II bis regula la competencia judicial internacional respecto a los menores, sin que establezca la distinción de edades que sí realiza el Convenio de la Haya de 19 de octubre de 1996 Relativo a la Competencia, la Ley Aplicable, el Reconocimiento, la Ejecución y la Cooperación en materia de Responsabilidad Parental y de Medidas de Protección de los Niños, que lo fija en su artículo 2 en los 18 años, o el Convenio de 25 de octubre de 1980 sobre los Aspectos Civiles de la Sustracción Internacional de Menores que lo fija en su artículo 4 en niños hasta 16 años. Por ello debemos aplicar la legislación de cada Estado, y considerar a nuestros efectos, a la hija de 17 de años como menor hasta que cumpla los 18.

${ }_{35}$ Sobre la oportunidad del criterio de la residencia habitual de los menores para determinar la competencia judicial internacional y los criterios a emplear, vid. J. CARRASCOSA GonZÁlez, "Litigación internacional, responsabilidad parental y foro de la residencia habitual del menor en un Estado miembro. Un estudio jurisprudencial", en A. Cebrián Salvat/ I. Lorente Martínez, Protección de menores y Derecho Internacional Privado, Granada, Comares, 2018, pp. 307 a 324. Sin ser objeto central de este trabajo, sí citaremos que la posición del Tribunal Supremo español sobre la materia de la residencia habitual en la competencia judicial internacional la podemos analizar en A. YBARRA Bores, "Competencia judicial internacional y divorcios transfronterizos ante el Tribunal Supremo", en A. L. Calvo Caravaca/ J. Carrascosa González, El Tribunal Supremo y el Derecho Internacional Privado, vol I., los autores, Murcia, 2019, pp. 191 a 196. 


\section{Acierto en la valoración de los Tribunales españoles}

\section{Correcta valoración del lugar de residencia habitual de las partes}

12. Los Tribunales españoles consideraron que la residencia habitual de los cónyuges y de los hijos en el momento de la presentación de la demanda estaba en España, y en esto acertaron plenamente. La sentencia de instancia, a la que se remite la de apelación en lo relativo a la materia de Derecho internacional privado, establece que la residencia habitual estaba en España donde la familia al completo, los padres y los dos hijos que dependían de ellos, habían fijado el domicilio familiar desde hacía años antes de producirse la ruptura en febrero de $2016^{36}$. Aciertan nuestros Tribunales en esta consideración, dándole la trascendencia a la vida diaria de las partes, al centro de sus intereses personales y familiares. El hecho de que vivan juntos y en el mismo lugar los padres y los dos hijos no independizados es esencial (hay una tercera hija del matrimonio ya independizada). En dicho domicilio desarrollan su día a día, en dicha localidad se forman los dos hijos ${ }^{37}$, y estos tienen sus relaciones personales habituales, ya que, como señala la resolución "desde hacía años" antes de la crisis familiar ya residían en España. Es cierto que hubiese sido deseable que hubiésemos tenido más datos sobre el momento de la llegada a España tras la boda y la convivencia en Francia, pero el hecho de que llevasen muchos años viviendo en España es suficiente para determinarlo. Otro motivo para concretar la residencia en España de la familia es que el demandado siga viviendo en España en el momento de dictarse la resolución de segunda instancia, y que el primer motivo de traslado de la madre y los hijos fue el de la precariedad económica en la que quedaron tras la ruptura y la necesidad de ir a vivir con una hermana que residía en Francia.

13. Los otros factores que vinculan a la familia con Francia no deben ser tenidos en cuenta en la valoración de su residencia habitual cuando se presenta la demanda. La residencia se ha de analizar en el momento de la crisis familiar ${ }^{38}$, y por ello, a estos efectos, no tiene ningún valor que los cónyuges se casasen en Francia, mucho tiempo antes, o que su matrimonio estuviese regulado por el régimen de separación de bienes del Código Civil francés. Incluso, aun siendo la nacionalidad común un criterio alternativo de localización de la competencia, sobre lo que incidiremos en el siguiente epígrafe, para la determinación de la residencia habitual lo esencial son sus vínculos y su vida diaria en dicho momento en un determinado territorio, pasando a un segundo plano la nacionalidad de ambas partes, tal y como ya ha dejado citado la Jurisprudencia del TJUE en relación a menores ${ }^{39}$, imponiéndose a la misma la presencia física en un determinado Estado miembro ${ }^{40}$. Así pues, consideramos acertado establecer la residencia habitual de la pareja y de la familia en España, y a partir de ahí valorar tanto la competencia judicial internacional como la ley aplicable.

14. Respecto a la hija de 17 años, alega el demandado en el acto del juicio, tras haber admitido la competencia inicialmente en la contestación a la demanda ${ }^{41}$, que ha cambiado de residencia habitual, y que en dicho momento vivía en Francia, y que por ello se debe cambiar la competencia judicial internacional por ser de los Tribunales franceses. Desarrollaremos este segundo aspecto en el epígrafe III.2, pero

${ }^{36} C f$. FJ $1^{\circ}$ de la resolución de instancia, p. 5.

${ }^{37} C f$. FJ $5^{\circ}$ de la sentencia de apelación, que señala que el hijo se formaba en una escuela de restauración en España, estudios que no pudo finalizar por no ser abonadas sus últimas cuotas.

${ }^{38} C f$. art. 8.1, última frase, “...en el momento en que se presenta el asunto ante el órgano jurisdiccional”. Al respecto, vid. A. L. Calvo Caravaca/ J. Carrascosa González, Derecho Internacional Privado, Vol. II, Madrid, $18^{\text {a }}$ edición, Comares, 2018 , p. 237.

${ }^{39} \mathrm{Vid}$. STJUE de 28 de junio de 2018, Caso HR, Asunto C-512/17, CLI:EU:C:2018:513. En el caso de una menor de muy corta edad (un año y 5 meses), con doble nacionalidad belga y polaca y empadronamiento en ambos países, con vida habitual y guardería en Bélgica, con visitas habituales a sus familiares polacos y mayor dominio del polaco por su corta edad, el TJUE estableció que la presencia física de la menor se imponía como criterio de valoración de la residencia habitual a la de su nacionalidad y la de sus abuelos.

40 Vid. STJUE de 17 de octubre de 2018, Caso UD, Asunto C-393/18, ECLI:EU:C:2018:835. En este supuesto se argumentaba que una menor que tenía padre británico y madre bangladesí y que había nacido en Bangladesh tenía residencia habitual en el Reino Unido, estableciendo el TJUE que una menor que nunca había residido en un Estado miembro no podía, en ningún caso, tener residencia habitual en el mismo.

${ }^{41} C f$. FJ $1^{\circ}$ de la resolución de instancia, p. 3. 
respecto a la residencia habitual de la menor, hemos de indicar que nos faltan datos concretos en el proceso para poder determinar que su residencia habitual debe estar fijada ya en el país vecino, en especial el de su voluntad futura de permanecer en él o no. Esto es así porque, tal y como hemos expuesto en el epígrafe II, la voluntad y la intención de permanencia en un determinado lugar es un elemento clave para determinar si ha fijado allí su residencia habitual o no. Se puede estar en un lugar una semana y ser la residencia habitual por la estabilidad futura en ella, y estar cuatro meses y no suponerlo por ser un lugar de paso ${ }^{42}$.

15. Sin embargo, de los datos del proceso podríamos intentar concretar una hipótesis. La Sentencia de segunda instancia indica que en el momento en el que surge el conflicto, dada su situación precaria, la madre se traslada a Francia a vivir con una hermana. Sin embargo, en el momento del dictado de la resolución los tres viven de alquiler en una vivienda que se han procurado, la menor va a una escuela y tiene los gastos típicos de una vida ordinaria de una joven de su edad, mutua médica, personales, médicos, o de actividades extraescolares, y el menor se forma en ciclos de restauración en Francia ${ }^{43}$. Sin conocer más datos, creemos que se puede considerar que existe cierta estabilidad en la vida actual de la menor en Francia, y que, por ello, con alguna duda, podríamos partir de la hipótesis futura, no confirmada, de que es posible que la menor tenga su residencia habitual en Francia. Sin embargo, debemos valorar también durante el resto de nuestro trabajo que podría ser absolutamente cierto que esta vida en Francia sea temporal y esté supeditada a obtener una mayor capacidad económica cuando finalice el proceso y reciba la pensión alimenticia mensual y en dicho momento volver a residir en España, que es el lugar en el que los dos hijos de la pareja y la madre habían vivido en los últimos años.

\section{Competencia judicial española y el cambio de residencia de la hija}

16. Los cónyuges son de nacionalidad francesa, residen en España e inician un procedimiento de divorcio contencioso ante los Tribunales españoles. Por ello, en aplicación del artículo 1.a del Reglamento Bruselas II bis entran dentro de su ámbito de aplicación, constituyendo el mismo el régimen aplicable para determinar la competencia judicial internacional de los tribunales españoles en las casusas de divorcio, desplazando, por ello a la LOPJ ${ }^{44}$. En la concreción de la competencia el artículo 3 establece, como expusimos en el epígrafe II.2, 7 foros alternativos, de entre los cuales el primero de los mismos es la residencia habitual de los cónyuges si es la misma. Ese foro coincide por su propia naturaleza con el del domicilio del demandado, que evidentemente es el mismo. Por ello, la aplicación de la demandante de dicha norma y su interposición de la demanda de divorcio ante los tribunales españoles es perfectamente ajustada a Derecho. En ese momento, la residencia de los menores que reclaman alimentos es también España, por lo que en aplicación del artículo 8 del Reglamento, la competencia para conocer la demanda en lo relativo a las medidas parentales es la misma, España. No estamos ante un caso de divergencia entre la residencia habitual de las partes en el momento de la demanda, que podría llevar a plantearnos si la competencia podría duplicarse o $\mathrm{no}^{45}$, sino en un cambio de residencia tras esta, lo que analizaremos más adelante en el presente epígrafe.

${ }^{42}$ Vid. Guía práctica de la Comisión Europea para la aplicación del Reglamento Bruselas II bis, p. 27, que señala claramente que; "Aunque el uso del adjetivo «habitual» podría indicar una necesidad de establecer cierta duración de la residencia antes de que esta se pueda calificar de «habitual», no se ha de excluir la posibilidad de que un menor adquiera su residencia habitual en un Estado miembro el mismo día de su llegada, o poco después". $C f$. http://publications.europa.eu/resource/cellar/ f7d39509-3f10-4ae2-b993-53ac6b9f93ed.0007.01/DOC_1.

${ }^{43} \mathrm{Cf}$. $\mathrm{FJ}^{\circ}$ y $5^{\circ}$ de la sentencia de apelación. Estos indicios nos acercan a la aplicación del principio de proximidad, esencial para determinar la residencia habitual. Al respecto, vid. R. LAFUENTE SÁnchez, "Hacia un sistema unitario europeo en materia de ley aplicable a las sucesiones internacionales", $C D T$, octubre 2013, Vol. 5, № 2, p. 361.

${ }^{44} \mathrm{Vid}$. J. C. Fernández Rozas/ S. SÁnchez Lorenzo, Derecho Internacional Privado, Pamplona, 9a edición, Civitas, 2016, P. 449. Sobre si el Reglamento se aplica a todos los divorcios internacionales o podría haber situaciones en las que se aplicarían otras normas, se pronuncia, defendiendo la primera postura, y a dicho trabajo nos remitimos, A. QuiÑones EsCÁMEZ, “¿Cuándo se aplica el Reglamento Bruselas II bis? El TJCE se pronuncia sobre su ámbito de aplicación”, RDCE, $\mathrm{n}^{\circ} 29$, mayo y agosto 2008, pp. 457 a 482.

${ }^{45}$ Ocurriría si los menores no residiesen en el mismo lugar que sus padres en el momento de la interposición de la demanda, casos habituales cuando hay extranjeros residiendo en España que no han podido reagrupar a sus hijos que siguen en sus países 
17. La demandante podría haber iniciado el procedimiento en Francia, en el foro de la nacionalidad común de las partes, pero decidió utilizar otro foro, el de la residencia habitual de los cónyuges, por otro lado, mucho más natural para ambas partes ${ }^{46}$. Por ello la alegación introducida por el demandado en la apelación ${ }^{47}$ en la que, tras no triunfar la de la primera instancia del cambio de residencia de la menor, afirmaba que la competencia debe ser de los Tribunales franceses por ser ambos de nacionalidad francesa, estaba predestinada desde un principio a fracasar. Siendo los foros alternativos, una vez iniciado el proceso, y siendo los tribunales españoles competentes y el lugar del domicilio del demandado, sin haber sumisión expresa a los Tribunales franceses, no es cierto que al estar ante una cuestión apreciable de oficio los Tribunales españoles deban declarar la competencia de Francia. España, donde la demandante inició el proceso, es competente para conocer el asunto, y por ello los Tribunales debían, como hicieron, desestimar la alegación del demandado recurrente. La misma podía tener como finalidad obviar la institución de la litispendencia si el demandado hubiese intentado iniciar la demanda en Francia una vez iniciado el proceso en España ${ }^{48}$.

18. Sin embargo, la alegación del cambio de domicilio de la menor una vez iniciado el procedimiento realizada en la primera instancia ${ }^{49}$ sí que tenía un cierto recorrido. La parte alegó en el acto del juicio que la menor había cambiado su domicilio, y que en dicho momento los tribunales franceses eran los competentes para conocer del procedimiento. En primer lugar diremos que en tal caso, los Tribunales franceses serían los competentes no para conocer de la demanda de divorcio, ante la que estamos, sino de las medidas relacionadas con la responsabilidad parental, las relaciones personales, visitas o comunicaciones entre padres e hija ${ }^{50}$. Pues bien, en esta alegación nos encontramos ante la figura de la perpetuatio fori. Según la misma, el principio general del Reglamento, respecto a los menores, en su artículo 8 , es que el tribunal que sea competente según su aplicación por ser el de la residencia habitual del menor, conserva su competencia incluso aunque el menor sea trasladado al extranjero durante el curso del litigio ${ }^{51}$. Este es el principio general, la competencia debe seguir siendo del Tribunal español, y debe ser replanteada si se modifica la residencia de la menor únicamente en determinados supuestos, considerados muy excepcionalmente, según lo establecido por los artículos 9, 10, 12 y 15 del Reglamento.

19. Para el presente supuesto debemos analizar con detenimiento el artículo 15 , toda vez que ni el 9, ni el 10 ni el 12, están pensados para un caso como el actual ${ }^{52}$. El artículo 15 prevé que un Tribunal

de origen a cargo de otros familiares. En dicha situación sería factible que se plantease contenciosamente un procedimiento de divorcio en España y otro de medidas respecto a los familiares en un tercer país. No podemos detenernos en este supuesto, por no ser objeto de la presente resolución. Al respecto, vid. I. Lorente Martínez, "Divorcio en España y menores con residencia habitual fuera de la Unión Europea”, en A. Cebrián SALVAT e I. LoREnTE MarTínez, Protección de menores y Derecho Internacional Privado, Granada, Comares, 2018, pp. 104 y 105.

${ }^{46}$ Tal y como señalamos, la familia había estado muchos años viviendo en España en el momento de la interposición de la demanda y España era el centro habitual de su vida e intereses personales y de parte de los económicos, toda vez que solo el padre tenía bienes y negocios propios en Francia. Además, seguía siendo el domicilio del demandado. En tal circunstancia España era el foro más natural para las partes.

${ }^{47} \mathrm{Cf}$. FJ $2^{\circ}$ de la sentencia de segunda instancia.

${ }^{48}$ O ciertamente también un fin dilatorio. La litispendencia en la materia está regulada en el artículo 19 del Reglamento Bruselas II bis. Al respecto de los efectos de la litispendencia, y la obligación de los Tribunales europeos de seguir el proceso en el Estado Miembro en el que en primer lugar se inició el proceso, con cita de jurisprudencia del TJUE que desarrolla estos efectos, vid. E. Pérez Vera, y otros, Derecho Internacional Privado, Madrid, 2a edición, UNED, 2016, pp. 154 a 158. Sobre las reformas legislativas europea y española de 2015 sobre la litispendencia, vid. C. RosendE VILLAR, "Litispendencia y conexidad internacionales y sus últimas reformas legislativas europea y española”, $A E D I P r$, t. XVI, 2016, pp. 347 a 374.

${ }^{49} \mathrm{Cf}$. $\mathrm{FJ} 1^{\circ}$ de la resolución de primera instancia, p 5.

${ }^{50}$ La responsabilidad parental, en palabras del TJUE, son "los derechos y obligaciones conferidos a una persona física o jurídica en virtud de una resolución judicial, por ministerio de la ley o por un acuerdo con efectos jurídicos, en relación con la persona o los bienes de un menor". Vid. STJUE de 27 de noviembre de 2007, Caso C, Asunto C-435/06, ECLI:EU:C:2007:714, párrafo 53.

${ }^{51}$ Vid. J. Carrascosa GonzÁlez, "Litigación internacional, responsabilidad parental y foro de la residencia habitual del menor en un Estado miembro. Un estudio jurisprudencial”, en A. Cebrián Salvat/ I. Lorente Martínez, Protección de menores y Derecho Internacional Privado, Granada, Comares, 2018, p. 320.

${ }_{52}$ Porque el 9 está previsto para los cambios legales de residencia habitual si el progenitor que ya tiene una resolución judicial de régimen visitas sigue residiendo en la residencia habitual, lo que no es el caso. En el artículo 10, se regula la sustracción de 
que conozca de un asunto puede declinar su competencia para conocerlo, de manera excepcional, si considera que los Tribunales de otro Estado Miembro están mejor situados para conocer del asunto. Y hemos indicado que dicha alegación hubiese tenido más visos de prosperar, aunque consideramos acertado que no lo haya hecho, toda vez que se daban alguna de las condiciones que establece el artículo para considerar que el tercer Estado tiene una vinculación especial, e incluso más de una. Una de las condiciones que quizás se cumplía era que este otro Estado se haya convertido en el de residencia habitual de la menor tras la presentación de la demanda procedimiento ${ }^{53}$. Otra, que sea el Estado del que es nacional la menor. La última, que fuera la residencia habitual un titular de la patria potestad ${ }^{54}$. Dicho artículo ha sido concretado por la Jurisprudencia del TJUE ${ }^{55}$ que ha establecido que el artículo se debe interpretar en el sentido de que, para poder estimar que un órgano jurisdiccional de otro Estado miembro con el que el menor tenga una vinculación especial está mejor situado, el órgano jurisdiccional competente del Estado miembro inicialmente competente debe cerciorarse, en primer lugar, de que la remisión del asunto a dicho órgano jurisdiccional puede aportar un valor añadido real y concreto al examen del asunto, habida cuenta, en particular, de las normas de procedimiento aplicables en ese otro Estado miembro. Y, en segundo lugar, para poder estimar que tal remisión responde al interés superior del menor, el órgano jurisdiccional competente de un Estado miembro debe cerciorarse, en particular, de que dicha remisión no pueda incidir negativamente en la situación del menor.

20. Así pues, en caso de modificación de la residencia habitual, esta debe ser interpretada con arreglo a los principios informadores de la materialización, la protección y el interés superior del menor, que dominan la orientación y función del Reglamento, penetrando en el problema de la determinación de la competencia internacional de autoridades y de la cooperación entre las mismas ${ }^{56}$. Y en tales circunstancias, estos requisitos jurisprudenciales son los que nos hacen decantarnos porque los Tribunales españoles tomaron la decisión correcta de seguir considerándose competentes ${ }^{57}$. Si bien se dan tres de las condiciones objetivas para poder declarar más convenientes los Tribunales de Francia, ninguna de los dos requisitos jurisprudenciales del TJUE se dan de forma indubitada. En primer lugar, ya vimos que la residencia habitual de la madre y de la demandada ha sido España durante muchos años previos al divorcio, y que el motivo que en primer lugar les llevó a cambiar de residencia fue la necesidad económica temporal en la que les dejaba el procedimiento de divorcio, por lo que su voluntad podría ser volver a España una vez esta necesidad acabe. Por otro lado, la madre no ha solicitado como hizo el padre, la declaración de competencia de los Tribunales franceses, lo que nos indica que no aprecia un valor añadido al cambio de competencia. Y esto es así quizás porque, dada la precariedad económica de la madre e hijos durante el procedimiento en comparación con la abundancia del patrimonio del padre, su interés es no paralizar el procedimiento para cambiar de tribunales competentes y finalizarlo cuanto antes. Por último, el domicilio del demandado, que será el obligado a abonar las altas pensiones alimen-

menores, lo que tampoco ocurre en este supuesto. $\mathrm{Y}$ en el 12 la posibilidad de unificar la competencia entre los tribunales que conozcan del divorcio por la residencia de los padres y las decisiones de la tutela parental si el hijo tiene otra residencia habitual en el momento de la interposición de la demanda, lo que ya vimos que no ocurre en el presente supuesto. Al respecto, vid. M. GuzMÁN PECES, "La competencia judicial en materia de nulidad, separación y divorcio; responsabilidad parental y sustracción civil de menores en el Derecho internacional privado español", en M. GuZMÁn Zapater/ M. Herranz Ballesteros (directoras), Crisis matrimoniales internacionales y sus efectos. Derecho español y de la Unión Europea, Valencia, Tirant lo Blanch, 2018, pp. 257 a 261.

${ }_{53}$ Decimos que quizás se cumple porque ya dejamos citado en el epígrafe III.1 que no podemos asegurar al $100 \%$ que la residencia habitual de la menor haya cambiado definitivamente a Francia.

${ }^{54} \mathrm{El}$ artículo establece un procedimiento a instancia de parte, o de oficio o del órgano judicial del tercer Estado, en el que, al menos con el consentimiento de una de las partes, el órgano que lo conoce debe suspender el procedimiento, y solicitar al órgano mejor situado que ejerza su competencia que se declare competente en el plazo de seis semanas. Sobre el proceso puede consultarse la Guía práctica de la Comisión Europea para la aplicación del Reglamento Bruselas II bis, anteriormente ya citada, pp. 34 a 35.

55 Vid. STJUE de 26 de octubre de 2016, Caso D, Asunto C-428/16, ECLI:EU:C:2016:819, párrafo 61.

$56 \mathrm{Vid}$. J. J. VARA PARRA, "El interés del menor en los foros de competencia judicial para las acciones de responsabilidad parental en el Reglamento (CE) núm. 2201/2003”, REDI, vol. LXIII (2006) 2, pp. 797 a 820, p. 815. El autor realiza en el citado trabajo un valioso y muy detenido análisis de la perpetuatio iurisdiccionis que no podemos afrontar en nuestro estudio por motivos de objeto del mismo, análisis al que nos remitimos para una mayor profundidad.

${ }^{57}$ Ciertamente sin aplicar el artículo 15 , que no es citado en el FJ $1^{\circ}$ de la detallada y muy trabajada sentencia de instancia, pero sí resolviendo finalmente acorde a sus requisitos. 
ticias y compensatorias establecidas en las resoluciones, es España, por lo que a las beneficiadas por ellas les interesa, de cara a una posible ejecución y falta de pago del demandado, ejecutar una resolución española del mismo lugar del domicilio del demandado ${ }^{58}$. Por todo lo referido no se puede alegar que el cambio de competencia respondiese al interés de la menor ni que le otorgase valor añadido al examen del asunto y que por ello procediese declarar la competencia de los Tribunales franceses. En relación a la reclamación de alimentos, el Reglamento $4 / 2009^{59}$ no recoge ninguna previsión de cambio de competencia por cambio de residencia de la acreedora posterior a la interposición de la demanda similar al artículo 15 del Reglamento Bruselas II bis, por lo que no procede desarrollar ningún análisis al respecto.

\section{La ley aplicable, la española, sistema plurilegislativo}

21. Una vez establecida la competencia de los Tribunales españoles, en la determinación de la ley aplicable a las causas y motivos del divorcio nos encontramos en el ámbito de aplicación del Reglamento Roma III, por lo establecido en su artículo 1 cuando prevé que se aplicará en las situaciones que impliquen un conflicto de leyes, al divorcio y a la separación judicial como la actual, en la que se divorcian en España dos ciudadanos franceses, aplicación en la que acierta la resolución de instancia ${ }^{60}$. Por ello, a las causas del divorcio se le aplicó la legislación española, toda vez que era la residencia habitual de las partes en el momento en el que se interpuso la demanda. Sin embargo, en el resto de materias resueltas por los tribunales en la crisis familiar, dada la exclusión de su ámbito de aplicación de lo regulado por el Reglamento Roma III de las materias externas a las causas del divorcio hubiese sido oportuna algo más de concreción por parte de los Juzgados respecto a la normativa aplicada ${ }^{61}$. En relación a la responsabilidad parental, no cita la resolución de instancia la norma de conflicto que la regula en España ${ }^{62}$, el Convenio de la Haya de 19 de octubre de 1996 relativo a la Competencia, la Ley Aplicable, el Reconocimiento, la Ejecución y la Cooperación en materia de Responsabilidad Parental y de Medidas de Protección de los Niños. Este instrumento establece en los artículos 16 y siguientes que la ley aplicable será la de la residencia habitual de los menores. Los tribunales han aplicado la ley española, por lo que entendemos que han optado por aplicar la residencia habitual que tenían confirmada en el momento de interposición de la demanda la española, ya que no han considerado que la residencia posterior en Francia pueda constituirse como residencia habitual con los criterios que establece el Derecho europeo, por la falta de acreditación de la voluntad de constituirla como centro habitual de vida e intereses de las peticionarias. Todo lo contrario sería un error al no considerar el cambio de residencia habitual de los menores ${ }^{63}$.

${ }^{58} \mathrm{Si}$ bien el principio general del Derecho europeo es el de reconocimiento inmediato de las resoluciones judiciales, el principio de economía procesal aconseja ejecutar una sentencia en el mismo Estado en el que se dicta, más con las elevadas cuantías mensuales que debía abonar el demandado en ambos conceptos.

${ }^{59}$ Reglamento (CE) no 4/2009 del Consejo de 18 de diciembre de 2008 relativo a la competencia judicial, la ley aplicable, el reconocimiento y la ejecución de resoluciones y la cooperación en materia de obligaciones de alimentos, DOUE L7/1 de 10 de enero de 2009.

${ }^{60} \mathrm{Cf}$. FJ $2^{\circ}$ de la Sentencia de instancia. Damos por hecho que ambas partes han designado en sus escritos de demanda y oposición a la misma como la ley aplicable al procedimiento la ley española, y de ahí su referencia al artículo 5 y al acuerdo de ambas en esta determinación. En caso contrario deberían haberse referido al artículo 8 del mismo texto legal.

${ }^{61}$ El artículo 2 excluye de su ámbito de aplicación cuestiones como las consecuencias del matrimonio a efectos patrimoniales, la responsabilidad parental o las obligaciones de alimentos, también resueltas en el procedimiento analizado.

${ }^{62}$ Esta no es una materia que en todos los supuestos sea correctamente aplicada por los tribunales españoles. Al respecto, vid. S. Adroher Biosca, "Derecho aplicable a la resolución de las crisis matrimoniales y a la responsabilidad parental", en M. Guzmán Zapater y M. Herránz Ballesteros, Crisis matrimoniales internacionales, y sus efectos. Derecho español y de la Unión Europea, Valencia, Tirant lo Blanch, 2018, p. 369. Sobre la complejidad que provoca esta fragmentación legislativa, vid. M. Herranz Ballesteros, "Régimen Jurídico de las crisis matrimoniales internacionales y derecho aplicable: el Reglamento (UE) $n^{\circ}$ 1259/2010 del Consejo de 20 de diciembre de 2010, por el que se establece una cooperación reforzada en el ámbito d dela ley aplicable al divorcio y a la separación judicial", $R D U E, \mathrm{n}^{\circ} 22$ - enero-junio 2012, pp. 47 y 48.

${ }^{63} \mathrm{El}$ artículo 17 del Convenio señala que "La responsabilidad parental se rige por la ley del Estado de la residencia habitual del niño. En caso de cambio de la residencia habitual del niño, se rige por la ley del Estado de la nueva residencia habitual". Como citábamos, entendemos que los tribunales españoles o no han reparado en el contenido del último inciso del artículo, en cuyo caso habrían incurrido en un claro error y deberían haber aplicado la legislación francesa para determinar las medidas 
22. Sí que acertó la juzgadora de instancia cuando, en la determinación de la ley aplicable a las reclamaciones de alimentos de los hijos y de la esposa establece que las mismas están reguladas por el Reglamento europeo $n^{\circ} 4 / 2009$ de alimentos ${ }^{64}$ que nos remite al Protocolo de La Haya de 2007, acudiendo a la ley de la residencia habitual de los acreedores para regular dicha petición. También establece que la ley aplicable es la ley española, y al respecto debemos hacer la misma consideración que la del párrafo anterior respecto a que es posible que los Tribunales hayan considerado que los acreedores no habían cambiado de residencia habitual toda vez que la francesa tenía carácter provisional ${ }^{65}$.

23. Por último, y en relación a la aparición del derecho interregional, siendo la legislación española aplicable, el principio genérico del Reglamento Roma $\mathrm{III}^{66}$ establece que teniendo España unidades territoriales con Derecho objetivo propio en materia de divorcio y separación, la misma se considera como un Estado a los efectos de la determinación de la ley aplicable, y por ello, se debe aplicar también en su respectivo ámbito, el Derecho Catalán. No teniendo el Derecho catalán disposiciones normativas específicas sobre casusas de divorcio, a las mismas se le aplicó correctamente el Código Civil español y el Código de Familia catalán a las medidas posteriores al divorcio, tanto en el caso de la resolución de instancia como la de apelación.

\section{Derecho internacional privado, argumentación y cuestiones terminológicas, la importancia de la exactitud}

\section{El ámbito de aplicación, lo que abunda a veces daña}

24. No queremos finalizar nuestro comentario a las resoluciones analizadas sin citar, aunque sea brevemente y a modo de reflexión general, la necesidad de concreción y exactitud en la argumentación y de la corrección del uso terminológico, tan necesario en todas las ramas del derecho, pero que es especialmente destacable en el Derecho internacional privado, por su complejidad técnica. Y para muestra dejaremos aquí algunas situaciones que se dieron en el presente procedimiento. En cuanto al régimen jurídico que resuelve el procedimiento, en el momento en el que el supuesto se encuentra dentro del ámbito de aplicación del Derecho europeo, los principios de primacía y de aplicación inmediata y directa de los mismos motivan que estos se impongan a la normativa española, que queda subordinada a las normas europeas ${ }^{67}$, y que no deberá ser aplicada, ni citada, so pena de confundir al lector de la resolución. Es un error demasiado común en nuestros Tribunales señalar que a un supuesto de divorcio internacional se le aplica el Reglamento Bruselas II bis y también, conjuntamente, la LOPJ ${ }^{68}$. En este caso, lo que abunda daña, porque no es cierto y es técnicamente incorrecto que la competencia sea esa porque lo señalan las dos normas. No procede citar todas las normas porque refuerce el argumento porque prevee los mismos

respeto a la menor, o han considerado no consolidado el cambio de residencia habitual, por las dudas que antes expresamos al respecto sobre su carácter de lugar del centro de los intereses de los mismos.

${ }^{64}$ Reglamento (CE) no 4/2009 del Consejo de 18 de diciembre de 2008 relativo a la competencia judicial, la ley aplicable, el reconocimiento y la ejecución de resoluciones y la cooperación en materia de obligaciones de alimentos, DOUE L7/1 de 10 de enero de 2009.

${ }^{65}$ Toda vez que el artículo 3.2 del Protocolo también establece que, en caso de cambio de la residencia habitual del acreedor, se aplicará la ley del Estado de la nueva residencia habitual desde el momento en que se produce el cambio. Si esto no fuese así, y los juzgadores valorasen la existencia de una nueva residencia habitual, debería haber sido la ley francesa la que regulase los alimentos reclamados.

${ }^{66}$ Cf. art. 14 del Reglamento. Vid. A. L. Calvo Caravaca/ J. Carrascosa González, Derecho Internacional Privado, Vol. II, op. cit., p. 284.

${ }^{67}$ Al respecto, vid. M. GuZmán Peces, “La competencia judicial en materia de nulidad, separación y divorcio...." op. cit., p. 250, que señala incluso que la LOPJ se aplicará únicamente en supuestos excepcionales, siendo la regla habitual la de que se aplique el Reglamento Bruselas II bis, y solo el Reglamento, no la LOPJ.

${ }^{68} \mathrm{Vid}$. A. QuiÑones EsCÁmez, “¿Cuándo se aplica...? op. cit., p. 465, en la que señala otro supuesto como ejemplo en el que la Audiencia Provincial de Barcelona también cita ambas normas, e incluso en primer lugar la LOPJ y subordinada a ella el Reglamento europeo. 
criterios para establecer los foros ${ }^{69}$. Todo lo contrario, debilitan el argumento, toda vez que parece confundirse si al supuesto se aplica la LOPJ o el Derecho europeo, y las consecuencias de las lecturas de ambas normas puede ser diferente, por ejemplo, en algo tan determinante como la concreción de la residencia habitual, ya que ya señalamos que el concepto es autónomo del Derecho europeo, diferenciado del concepto del mismo de cada derecho nacional.

25. Y este error, por otro lado, habitual, ocurrió tanto en la sentencia de instancia como en la de apelación. La sentencia de instancia afirmó que la competencia viene regulada por el Reglamento Bruselas II bis y por otro lado, por lo previsto en la $\mathrm{LOPJ}^{70}$. La sentencia de apelación afirmó que se aplica el Reglamento europeo en relación a la LOPJ ${ }^{71}$. Tampoco se puede señalar, tal y como afirma la sentencia de instancia, que la ley aplicable viene regulada por el Reglamento Roma III en relación al artículo 9.2 y 107 del Código Civil ${ }^{72}$. No se aplica en relación a esas normas. Tienen relación con ellas porque ambas regulan materias de Derecho internacional privado, y podrían ser aplicables a las distintas situaciones que se pueden resolver en un determinado supuesto, pero no se aplican una en relación a la otra. De hecho, si se aplican las normas internas es precisamente porque la norma europea ha dejado fuera de su ámbito de aplicación dicha materia, es decir, porque no se aplica relacionada con ella.

\section{El derecho aplicable, la importancia de la argumentación}

26. Otro error que se suele apreciar en ocasiones en nuestros Tribunales se produce en la forma de expresar la relación que existe entre dos normas, la europea, el Reglamento Roma III y la española, el artículo 107.2 del Código Civil, que regulan la ley aplicable a la separación y el divorcio internacionales, y el hecho de que la norma española fuese modificada en 2015 señalando que en estos supuestos se aplica la normativa europea. Esta regulación provoca que, como ha ocurrido en este supuesto, los tribunales expresen de forma absolutamente inadecuada cuál es la relación entre ambas normas. La resolución de instancia afirma ${ }^{73}$ que, toda vez que el artículo 107.2 establece que el divorcio se regirá por las normas de la Unión Europea, se debe aplicar al supuesto el Reglamento Roma III. Y esta argumentación es claramente errónea. El Reglamento Roma III, aplicable con preferencia a la legislación española por las ya citadas primacía y efecto inmediato y directo, establece en su artículo 4 que es de aplicación universal, erga homnes, y se aplicará, aunque la ley designada sea de un Estado extraño a la UE. Esta regulación ha provocado, de hecho, no solo que no sea cierto que el Reglamento europeo se aplique gracias a que así lo establece el artículo 107, sino que este haya quedado sustituido, desplazado, e incluso algunos autores señalan que prácticamente derogado, por el ámbito de aplicación de Roma III ${ }^{74}$. Así pues, dicha argumentación es claramente inadecuada.

\section{Control de oficio de la competencia y la perpetuatio fori}

27. En último lugar apuntaremos dos aspectos ya tratados en el desarrollo del presente estudio y que no fueron correctamente reflejados en las sentencias analizadas. El primero es el de la apreciación de la competencia judicial internacional. Señala la demandada y la Audiencia Provincial en su resolución $^{75}$, que la competencia debe ser correctamente apreciada por los Tribunales incluso con alegación posterior a la contestación a la demanda con la genérica argumentación del principio iura novit curia,

\footnotetext{
${ }^{69}$ Ya señalamos en la nota 19 la inspiración del legislador español en 2015 en el Derecho europeo para la modificación de la LOPJ.

${ }^{70} \mathrm{Cf}$. FJ $1^{\circ}$ de la resolución de instancia, p. 4. La resolución transcribe literalmente ambas normas, pero primero la LOPJ y tras ello el Reglamento europeo.

${ }^{71} C f$. FJ $2^{\circ}$ de la sentencia de apelación.

${ }^{72} C f$. FJ $1^{\circ}$ de la resolución de instancia, p. 5.

${ }^{73} C f$. FJ $2^{\circ}$ de la resolución de instancia, p. 7.

${ }^{74} \mathrm{Vid}$. S. Adroher Biosca, "Derecho aplicable a la resolución de las crisis matrimoniales...”, op. cit. pp. 366 y 367.

${ }^{75} \mathrm{Cf}$. $\mathrm{FJ} 2^{\circ}$ de la resolución de apelación.
} 
o a la específica de la cuestión de orden público. Sin embargo, la sentencia de primera instancia afirmó con gran acierto que estamos ante una cuestión apreciable de oficio ${ }^{76}$. La institución ante la que estamos en la apreciación de la competencia judicial internacional es su apreciación de oficio por nuestros Tribunales en el momento en el que lo adviertan, sea cual sea, tal y como lo establece el artículo 38 de la LEC en el ámbito interno ${ }^{77} \mathrm{y}$, en la competencia judicial internacional en divorcio y separación en el artículo 17 del Reglamento Bruselas II bis ${ }^{78}$. Es importante que lo citemos correctamente toda vez que acudir a la institución del orden público en el Derecho internacional privado puede provocar confusión respecto a la denegación de ejecución de las resoluciones por motivos de orden público, o al control de la competencia de los órganos jurisdiccionales de origen, prohibido, por ejemplo, por el artículo 24 del Reglamento Bruselas II bis.

28. Por último, señalaremos con brevedad que el mantenimiento de la competencia en este procedimiento se producía por la perpetuatio fori, por el mantenimiento de la competencia de los Tribunales competentes incluso tras el traslado de la menor salvo que fuese aplicable el artículo 15 del Reglamento, lo que como antes señalamos, no se producía en el presente procedimiento, sin que ninguna de las resoluciones analizadas haya citado ni el artículo aplicado ni la institución jurídica que provoca la final inadmisión de la alegación del demandado.

\section{Conclusiones, algo más que la residencia habitual}

29. La Sentencia de la Audiencia Provincial de Girona de 24 de julio de 2019, y la del Juzgado de Primera Instancia e Instrucción de Figueras de 18 de diciembre de 2018, aciertan en la determinación de la competencia judicial internacional y la ley aplicable en un procedimiento en el que las partes tenían estrechos vínculos con dos Estados. La residencia habitual de la pareja y de sus hijos estaba en España, toda vez que aquí tenían su vivienda familiar y desarrollaban sus actividades cotidianas como familia, estudios, amistades y trabajo, y la demandante obvió la nacionalidad común francesa y escogió correctamente los tribunales españoles como competentes dentro de los foros alternativos del artículo 3 del Reglamento Bruselas II bis.

30. Los cónyuges eran franceses, se casaron en Francia y su matrimonio estaba regulado por la separación de bienes del Código Civil Francés, pero estos criterios decayeron respecto a la trascendencia del lugar del centro de vida e intereses comunes de la familia en el momento de la crisis familiar, situado en España y que por ello debía ser considerada como su residencia habitual, criterio principal en el Derecho europeo de familia para definir la competencia judicial internacional. El traslado de la menor a Francia tras la interposición de la demanda no varía esta competencia en aplicación del artículo 15 del Reglamento Bruselas II bis según lo ha concretado la Jurisprudencia del TJUE, toda vez que no quedó acreditado que dicho cambio de Estado competente supusiese un valor añadido real en el proceso y respondiese al interés superior de la menor.

31. Las sentencias también nos demuestran la importancia de la correcta argumentación y la terminología empleada en Derecho internacional privado. Si el ámbito de aplicación de un Reglamento determina que debe ser aplicado por los Tribunales españoles, los principios de primacía y aplicación directa del Derecho europeo señalan que no se deben citar, para mayor abundamiento, las normas españolas, en

\footnotetext{
${ }^{76} \mathrm{Cf}$. FJ $1^{\circ}$ de la resolución de instancia, p. 5.

${ }^{77} \mathrm{Vid}$. al respecto a E. Castellanos Ruiz y J. Rodríguez Rodrigo, "Comentarios a la Ley de Enjuiciamiento Civil”, InDret, no 3, julio, 2016, pp. 16 a 18, o a A. Rodríguez Benot (Director), Derecho Internacional Privado, op. cit. p. 48.

${ }_{78}$ Que establece que el órgano jurisdiccional de un Estado miembro ante el que se inicie un procedimiento respecto del cual el presente Reglamento no establezca su competencia y del que sea competente en virtud del mismo un órgano jurisdiccional de otro Estado miembro, se declarará de oficio incompetente. Al respecto, vid. J. C. Fernández Rozas/ S. Sánchez Lorenzo, Derecho Internacional Privado, op. cit. p. 98, o a P. OreJudo Prieto de los Mozos, "Diez años de aplicación e interpretación del Reglamento Bruselas I bis...”, op. cit., p. 24.
} 
relación o conjuntamente con el mismo, como ocurre habitualmente en nuestro foro. Por otro lado, sobre la competencia, lo correcto es señalar que la misma es apreciable de oficio por nuestros Tribunales, y no acudir a la genérica argumentación del principio iura novit curia, o a la específica de la cuestión de orden público, relacionada con el derecho aplicable, lo que puede provocar confusión. Por último, ninguna de las resoluciones citó expresamente el mantenimiento de la competencia de los Tribunales españoles por no ser aplicable el artículo 15 del Reglamento Bruselas II bis, verdadera causa de la misma. 